\title{
Mathematical Modelling and Experimental Evaluation of Electrostatic Sensor Arrays for the Flow Measurement of Fine Particles in a Square-shaped Pipe
}

\author{
Shuai Zhang, Yong Yan, Fellow, IEEE, Xiangchen Qian, and Yonghui Hu
}

\begin{abstract}
Square-shaped pneumatic conveying pipes are used in some industrial processes such as fuel injection systems in coal-fired power plants and circulating fluidized beds. However, little research has been conducted to characterise the gas-solid two-phase flow in a square-shaped pneumatic conveying pipe. This paper presents mathematical modelling and experimental assessment of novel non-restrictive electrostatic sensor arrays for the measurement of pulverised fuel flow in a square-shaped pipe. The sensor arrays consist of twelve pairs of strip-shaped electrodes, which are uniformly embedded in the four flat pipe walls. An analytical mathematical model of the sensor arrays is established and the induced charge and currents of different electrodes due to a point charge are then derived based on the model. Experimental tests were conducted on a $54 \mathrm{~mm}$ square-shaped pipe section of a pneumatic conveyor test rig under a range of flow conditions. The fuel velocity profile over the whole cross-section of the pipe is measured. Mathematical modelling and experimental results demonstrate that the proposed non-restrictive electrostatic sensor arrays are capable of characterising the local pulverised fuel flow in a square-shaped pneumatic conveying pipe.

Index Terms-electrostatic sensor, square-shaped pipe, mathematical modelling, velocity profile, pulverised fuel.
\end{abstract}

\section{INTRODUCTION}

$\mathrm{I}^{\mathrm{N}}$ coal-fired power plants, pulverised fuel is pneumatically conveyed towards a matrix of burners via a primary air pipe network. The fundamental parameters of gas-fuel flow, such as the particle velocity, solid concentration and flow stability, play an important role in improving thermal efficiency and reducing pollutant emissions. Due to the advantages of simple structure and easy installation, square-shaped pneumatic conveying pipes are applied in some industrial processes such as circulating fluidized beds [1] and some thermal power plants in certain countries, e.g., Germany and China. The characteristics of gas-solid flow in a square-shaped pipeline are of considerable engineering importance.

This work was supported in part by the Chinese Ministry of Education under Grant B13009, and in part by the Fundamental Research Funds for the Central Universities under Grant 2014XS46 and Grant 2016MS40. S. Zhang would also like to thank the Beijing Municipal Commission of Education for supporting the work

S. Zhang, X. Qian, and Y. Hu are with the School of Control and Computer Engineering, North China Electric Power University, Beijing 102206, China.

Y. Yan is with the School of Engineering and Digital Arts, University of Kent, Canterbury, Kent CT2 7NT, U.K, and also with the School of Control and Computer Engineering, North China Electric Power University, Beijing 102206, China (e-mail: y.yan@kent.ac.uk).
Owing to the existence of the time-varying interphase force between the air and solid phases, the dynamics of the flow in pneumatic conveying pipelines is extremely complex. Therefore, most of existing methods, such as thermal [2, 3], acoustic [4], capacitive [1], mechanical [5] and optical techniques [6], have shown fundamental limitations in their applications for measuring gas-solid flows, especially when the particles are conveyed in a dilute phase flow [7]. Due to the advantages of structural simplicity, robustness and low cost, electrostatic sensors outperform other techniques and thus are extensively utilised in gas-solid flow measurement. Electrostatic sensors with ring [8], [9], arc [9], [10], bar [11] or other shaped electrodes [12] have been used in circular pipelines. However, little research has been conducted on the application of electrostatic sensors on square-shaped pneumatic conveying pipes [13]. Murnane et al. [14] proposed a physical model of the square-shaped electrode and derived the distribution of induced charge density on the electrode. Peng et al. [15] developed a mathematical model of the square-shaped electrode and studied its characteristics both theoretically and experimentally, such as the sensing field distribution and spatial filtering characteristics. Turbulent duct flows in square-shaped duct were studied by Yao et al. [16], [17] through large eddy simulation in a large range of high Reynolds numbers. The simulation results indicate that the dynamic characteristics of particles close to the four sharp corners of the pipe are more complex than those in circular pipes.

Although the square-shaped electrodes proposed by Murnane et al. [14] and Peng et al. [15] can be utilised in square-shaped pneumatic conveying pipes, such electrodes do not provide local information about the particle flow due to their structural limitation of the full-square shape. In order to characterise the local particle flow in a square-shaped pneumatic conveying pipe, novel non-restrictive electrostatic sensor arrays with distributed, strip-shaped electrodes are designed, constructed and evaluated. A mathematical model of the sensor arrays is also established, from which the induced charge and the resulting currents on the electrodes due to a moving point charge are derived. The spatial sensitivity distribution over the pipe cross section is determined and illustrated. Based on the structure of the sensor arrays, a data fusion method is proposed to measure the particle velocity profile over the cross-section of the square-shaped pipe. 


\section{Design And Modelling Of Electrostatic Sensor ARRAYS}

\section{A. Design of Electrostatic Sensor Arrays}

Because of the friction between particles and air, collisions between particles, and impacts between particles and pipe wall, pulverised fuel particles are electrostatically charged when being conveyed in a pipeline. As the charged particles pass an electrode, a certain amount of electrostatic charge is induced on its surface. In order to obtain the velocity profile of pulverised fuel over the cross-section of a square-shaped pipe, the sensor arrays with distributed strip-shaped electrodes are designed, as shown in Fig. 1.

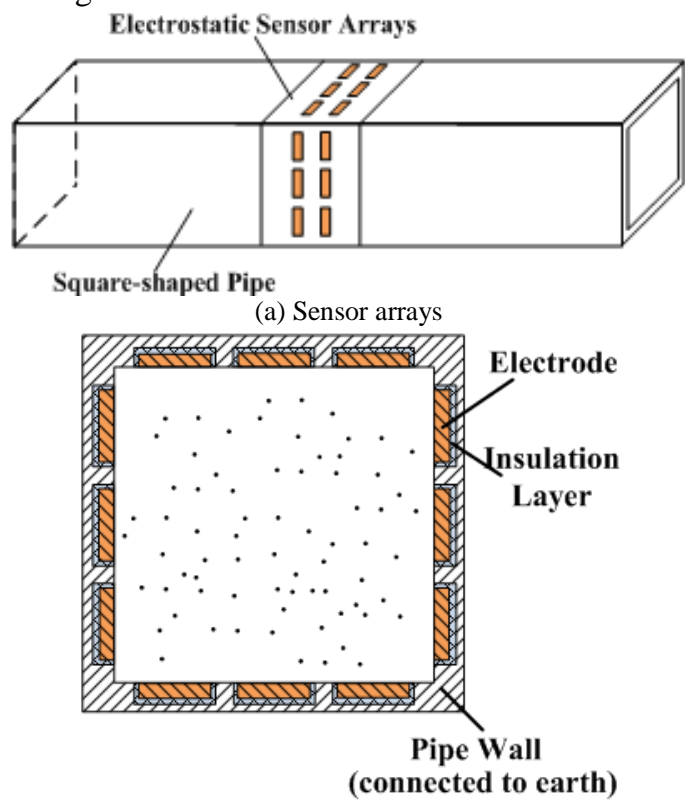

(b) Cross section of the sensor arrays and square pipe

Fig. 1. Physical structure of the electrostatic sensor arrays.

As shown in Fig. 1(a), a total of 24 strip-shaped electrodes (4 $\times 3$ pairs) are uniformly embedded in the four flat pipe walls. Fig. 1(b) illustrates the cross section of the sensor arrays. Three identical pairs of electrodes, shielded by the grounded pipe and insulated from the wall through a papery insulation layer, are embedded on one side wall. Compared to the square-shaped electrodes in previous studies [14], [15], the developed sensor arrays have a higher spatial resolution [10]. Three pairs of electrodes on each side of the pipe section are used to measure the localized information about the particles in the corners and away from the corners in this study. An excessively high number of electrode pairs on each side of the pipe section, though increasing the spatial resolution of the sensor, will entail significant computing power required, long signal processing time and slow system response. To have a higher sensitivity, the electrodes are mounted flush with the inner surface of the pipe walls and exposed to the particles. The centre-to-centre spacing between a corresponding pair of electrodes depends on the dimensions of the electrodes. Meanwhile, in order to suppress the noise from the environment, the signals from the electrodes are transmitted to the signal conditioning unit by co-axial cables.
The signals from the sensor arrays are amplified and filtered by a signal conditioning unit. Then the signals are sampled by a data acquisition (DAQ) card and processed by a host computer. The block diagram of the measurement system is shown in Fig. 2 .

\section{B. Mathematical Modelling of Electrostatic Sensor Arrays}

To gain an insight of the interactions between the charged particles and the electrostatic sensor arrays in square-shaped pipes and quantify the sensing characteristics of the arrays, a mathematical model of the sensor arrays is developed based on the theory of electrostatics together with the method of images [18]. The charged particles are the sources of the signals from the electrodes, and based on the principle of superposition, an ideal point charge passing the electrodes can be regarded as an impulse input to the sensing system [8]. It should be noted that, the signal from an electrostatic sensor contains both the induced charge (when charged particles pass the electrode) and the transferred charge (when contact occurs between charged particles and the electrode). However, only the induced charge is useful in the velocity profile measurement based on the cross correlation method, because of the low probability for a particle to contact the two electrodes successively in the dilute-phase pulverised fuel flow.

To calculate the induced charge on the electrodes, the electric field $E$ or the electric potential $\varphi$ near the surface of the electrode should be known [8], [14]. Owing to the symmetrical structure of the sensor arrays, the three adjacent electrodes, which are in the same cross-section plane and on one side of the wall, are analysed as an example in this study.

The electric field distribution for a point charge $+q$ near the three electrodes is illustrated in Fig. 3(a). The pipe wall is connected to earth. Compared to the dimensions of the point charge and electrodes, the inner wall of the pipe can be regarded as an infinite grounded conducting plane with zero potential. Because the point charge $+q$ will induce a certain amount of negative charge on the nearby surface of the conductors, the total potential of a given point is due in part to $+q$ directly, and in part to this induced charge on the nearby conductors [18]. In brief, the potential of the region above the wall and near the electrodes in the interior region is not $q / 4 \pi \varepsilon R$ ( $\varepsilon$ is the permittivity of the medium in the interior region of the pipe and $R$ is the distance between the point charge and the electrode). It is difficult to calculate the potential or the induced charge on one electrode accurately, when the induced charge on others is unknown.

From another perspective, the electric field produced by two opposite polarity point charges $(+q$ and $-q)$ is shown in Fig. 3(b). In the interior region, the electric field distribution in Fig. 3(a) and Fig. 3(b) are exactly the same. Based on the uniqueness theorem and the method of images [18], the three electrodes are supposed to be in the electric field generated by $+q$ and $-q$. 


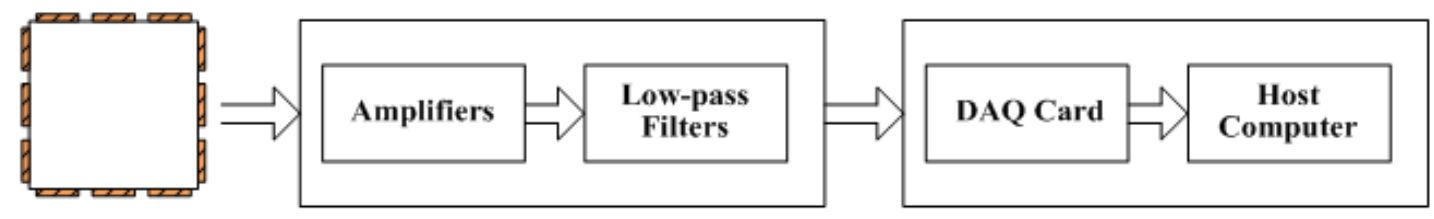

Sensor Arrays $\quad$ Signal Conditioning Unit Signal Processing Unit

Fig. 2. Block diagram of the measurement system.

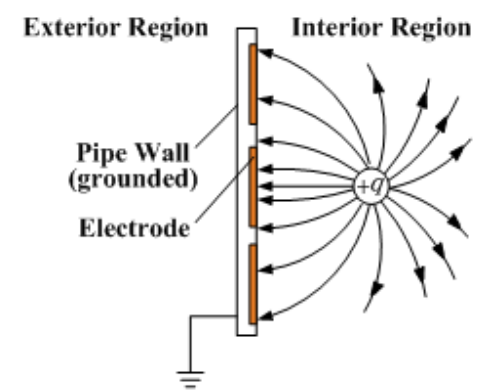

(a) Electric field distribution for a point charge Exterior Region Interior Region

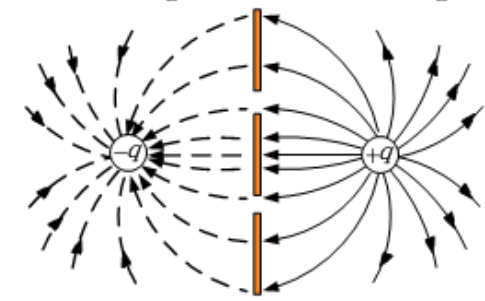

(b) Equivalent electrical field distribution generated by two point charges of opposite polarity

Fig. 3. Electric field distribution due to point charges.

The mathematical coordinate system of the sensor arrays is shown in Fig. 4. The median of the sensor electrode lies on the plane XOY and the origin is located at one corner of the square-shaped pipe. The three electrodes analysed in this paper is on the plane XOZ. This modelling is established with the following assumptions:

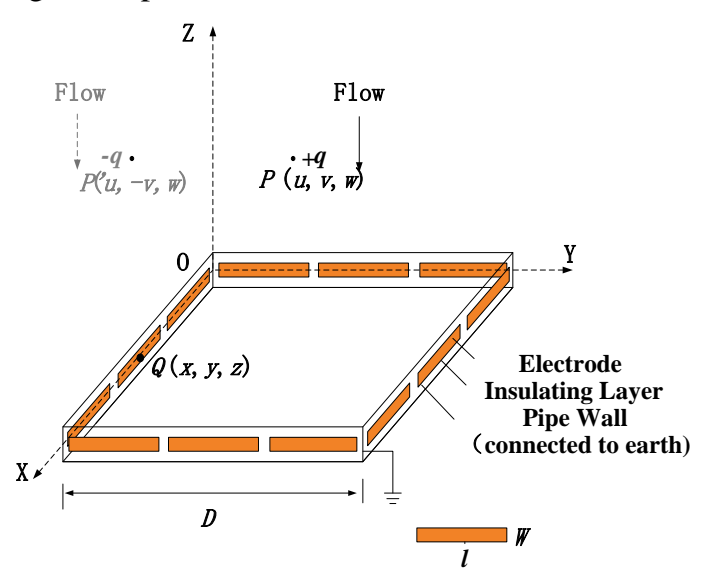

Fig. 4. Mathematical coordinate system for modelling the electrostatic sensor arrays.

(a) An ideal point charge $(P)$ with the electric quantity of $+q$ and its mirror point charge $\left(P^{\prime}\right)$ with the electric quantity of $-q$ are assumed to have axial velocity only.

(b) The geometric dimensions of the electrodes are $W$-width, $l$-length, $D$-length of inner side, and the thickness of the electrode is negligible.

(c) Influence of the thin insulating layers on the distribution of electric field in the sensing area is negligible. A given point $Q(x, y, z)$ is located on the right side of the $\mathrm{XOZ}$ plane and is in the electric field due to point charges $P(u, v, w)$ and $P^{\prime}(u,-v, w)$. The potential $\varphi$ at point $Q(x, y, z)$ is described as:

$$
\begin{aligned}
& \varphi=\frac{q}{4 \pi \varepsilon|P Q|}+\frac{-q}{4 \pi \varepsilon\left|P^{\prime} Q\right|} \\
& =\frac{1}{4 \pi \varepsilon}\left(\frac{q}{\sqrt{(u-x)^{2}+(v-y)^{2}+(w-z)^{2}}}\right. \\
& \left.-\frac{q}{\sqrt{(u-x)^{2}+(-v-y)^{2}+(w-z)^{2}}}\right)
\end{aligned}
$$

Then the surface charge density $\sigma$ induced on the three electrodes is given by:

$$
\sigma=-\left.\varepsilon \frac{\partial \varphi}{\partial y}\right|_{y=0}=\frac{-q v}{2 \pi\left[(u-x)^{2}+v^{2}+(w-z)^{2}\right]^{3 / 2}}
$$

So the total induced charge $q^{\prime}$ on the electrode surface is determined by:

$$
q^{\prime}=\int_{a}^{b} \int_{-\frac{W}{2}}^{\frac{W}{2}} \sigma d x d z
$$

where $a, b$ are the coordinate values of the both ends of the electrode on $\mathrm{X}$-axis and $b-a=l . q^{\prime}$ is given by combining equations (2) and (3):

$$
\begin{aligned}
& q^{\prime}=\frac{-q}{2 \pi}\left\{\operatorname{sgn}\left(w-\frac{W}{2}\right) \arctan \left[\frac{\left|w-\frac{W}{2}\right|(u-b)}{v \sqrt{(u-b)^{2}+v^{2}+\left(w-\frac{W}{2}\right)^{2}}}\right]\right. \\
& -\operatorname{sgn}\left(w-\frac{W}{2}\right) \arctan \left[\frac{\left|w-\frac{W}{2}\right|(u-a)}{v \sqrt{(u-a)^{2}+v^{2}+\left(w-\frac{W}{2}\right)^{2}}}\right] \\
& -\operatorname{sgn}\left(w+\frac{W}{2}\right) \arctan \left[\frac{\left|w+\frac{W}{2}\right|(u-b)}{v \sqrt{(u-b)^{2}+v^{2}+\left(w+\frac{W}{2}\right)^{2}}}\right] \\
& \left.+\operatorname{sgn}\left(w+\frac{W}{2}\right) \arctan \left[\frac{\left|w+\frac{W}{2}\right|(u-a)}{v \sqrt{(u-a)^{2}+v^{2}+\left(w+\frac{W}{2}\right)^{2}}}\right]\right\}
\end{aligned}
$$

Equation (4) can be solved analytically. As expected, the induced charge of the electrode has opposite polarity compared with the moving point charge [8]. From this analytical 
modelling, it can be found that, as a point charge (with electric quantity of $+q$ ) passes the sensor arrays, the value of the induced charge on the electrode $q$ ' depends on the following factors: dimensions of the electrode $(W, l)$, the positions of the electrode $(a$ or $b)$ and the point charge $(u, v, w)$. To eliminate the geometric influence of the electrode, $D$ (the length of inner side) is taken as the basic dimensional unit in the sensing system. Then parameters $l, W, a, b, u, v$ and $w$ become dimensionless ones $\delta_{l}, \delta_{W}, \delta_{a}, \delta_{b}, \delta_{u}, \delta_{v}$ and $\delta_{w}$, respectively, i.e., $\delta_{l}=l / D, \delta_{W}=W / D, \delta_{a}=a / D, \delta_{b}=b / D, \delta_{u}=u / D$, $\delta_{v}=v / D, \delta_{w}=w / D$.

Substituting the above dimensionless parameters into equation (4), $q$ ' is rewritten as the following:

$$
q^{\prime}=\frac{-q}{2 \pi}\left\{\operatorname{sgn}\left(\delta_{w}-\frac{\delta_{W}}{2}\right) \arctan \left[\frac{\left|\delta_{w}-\frac{\delta_{W}}{2}\right|\left(\delta_{u}-\delta_{b}\right)}{\delta_{v} \sqrt{\left(\delta_{u}-\delta_{b}\right)^{2}+\delta_{v}^{2}+\left(\delta_{w}-\frac{\delta_{W}}{2}\right)^{2}}}\right]\right.
$$$$
-\operatorname{sgn}\left(\delta_{w}-\frac{\delta_{W}}{2}\right) \arctan \left[\frac{\left|\delta_{w}-\frac{\delta_{W}}{2}\right|\left(\delta_{u}-\delta_{a}\right)}{\delta_{v} \sqrt{\left(\delta_{u}-\delta_{a}\right)^{2}+\delta_{v}^{2}+\left(\delta_{w}-\frac{\delta_{W}}{2}\right)^{2}}}\right]
$$$$
-\operatorname{sgn}\left(\delta_{w}+\frac{\delta_{W}}{2}\right) \arctan \left[\frac{\left|\delta_{w}+\frac{\delta_{W}}{2}\right|\left(\delta_{u}-\delta_{b}\right)}{\delta_{v} \sqrt{\left(\delta_{u}-\delta_{b}\right)^{2}+\delta_{v}^{2}+\left(\delta_{w}+\frac{\delta_{W}}{2}\right)^{2}}}\right]
$$$$
\left.+\operatorname{sgn}\left(\delta_{w}+\frac{\delta_{W}}{2}\right) \arctan \left[\frac{\left|\delta_{w}+\frac{\delta_{W}}{2}\right|\left(\delta_{u}-\delta_{a}\right)}{\delta_{v} \sqrt{\left(\delta_{u}-\delta_{a}\right)^{2}+\delta_{v}^{2}+\left(\delta_{w}+\frac{\delta_{W}}{2}\right)^{2}}}\right]\right\}
$$

Then the actual output $I_{s}(t)$ of the electrode is given by:

$$
I_{s}(t)=\frac{d q^{\prime}}{d t}
$$

\section{Spatial Sensitivity}

Equation (4) indicates that the induced charge $q$ ' depends on the source charge, dimensions of the electrodes and the relative locations between the electrode and the particle. To study the sensing characteristics of the sensor arrays, the spatial sensitivity of the sensor arrays is defined as the absolute value of the induced charge $\left|q^{\prime}\right|$ on the electrode when a unity point charge is in the sensing zone of the pipe [8], [15]:

$$
S(u, v, w)=\left|\frac{q^{\prime}(u, v, w)}{q}\right|
$$

The dimensionless parameters of the electrode, $\delta_{l}$ and $\delta_{W}$, are set as $\delta_{l}=5 / 18, \delta_{W}=1 / 18$, respectively, in this study. Combining equations (5) and (7) yields the spatial sensitivity distribution of the three strip-shaped electrodes on the XOY plane, as shown in Fig. 5.

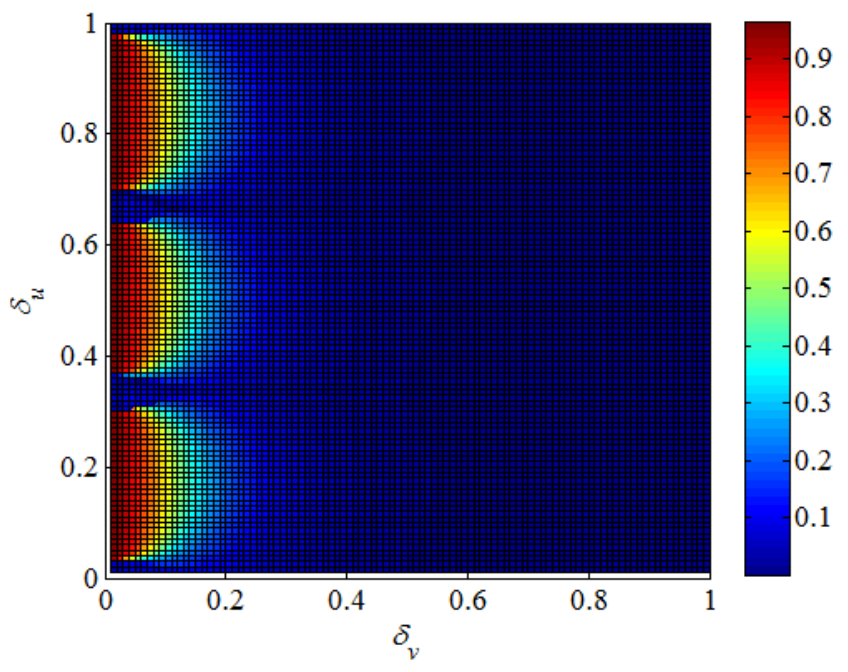

Fig. 5. Spatial sensitivity distribution over the XOY plane.

It can be seen that the strip-shaped electrode is more sensitive to particles closer to it. This trend demonstrates that the electrostatic sensors with distributed strip-shaped electrodes can be used to obtain local information about the particle flow in a square-shaped pneumatic conveying pipe.

\section{Induced Charge and Current of Electrostatic Sensor Arrays}

Based on the mathematical modelling presented above, typical examples of the induced charge $q^{\prime}$ and current $I_{s}(t)$ of the three electrodes as a particle (electric quantity $+q$ ) travels along different paths in the pipe with a constant velocity are shown in Fig. 6. The travelling path of the particle in Fig. 6(a) is $\delta_{u}=1 / 6, \delta_{v}=1 / 2$ and the path in Fig. $6(\mathrm{~b})$ is $\delta_{u}=1 / 2, \delta_{v}=1 / 2$.

As illustrated in Fig. 6, when the particle passes the electrodes, opposite polarity charge and the corresponding currents are induced on the electrodes. If the velocity of the particle and the dimensions of the electrode are constant, the value of the induced charge (or current) depends on the relative

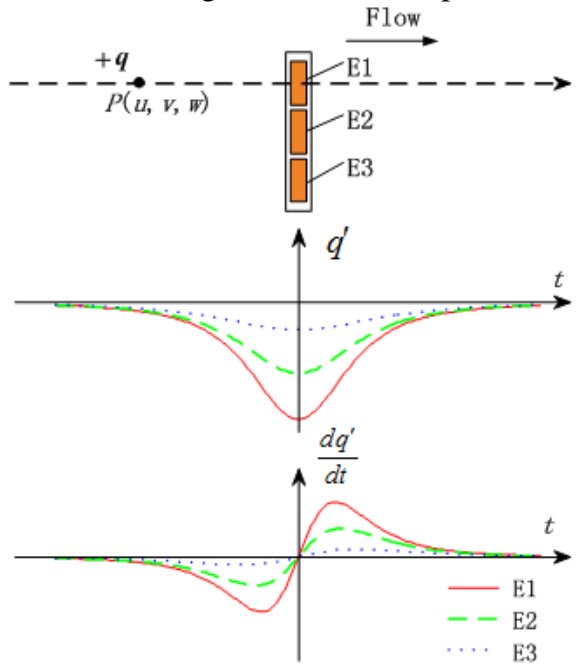

(a) $\delta_{u}=1 / 6, \delta_{v}=1 / 2$ 

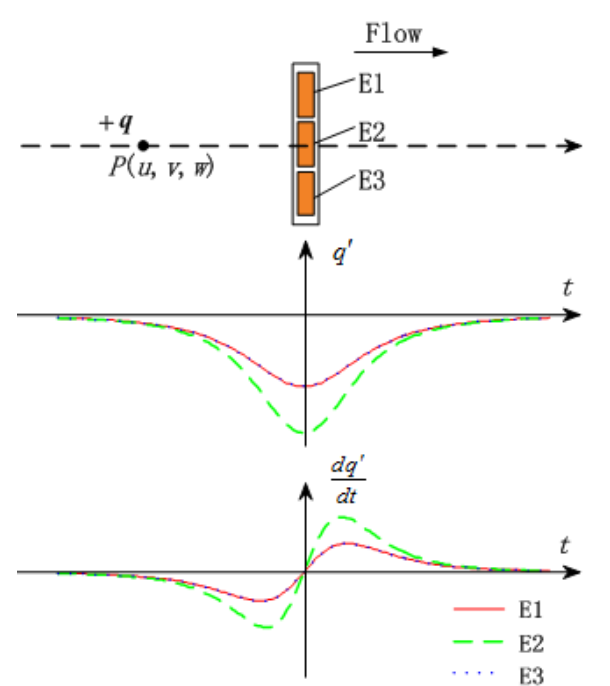

(b) $\delta_{u}=1 / 2, \delta_{v}=1 / 2$

Fig. 6. Typical examples of the induced charge and current on the electrodes when a particle travels along different paths in the pipe.

location of the electrode and the particle, i.e., the closer the particle to the electrode, the more induced charge or higher current will be induced. For example, the electrodes E1 and E3 are symmetrical about the moving particle $P$ in Fig. 6(b); therefore, the induced charge and current on electrode E1 are the same as those on electrode E3. These trends are in agreement with the spatial sensitivity distribution of an electrostatic electrode and the earlier research [8], [14], [15], which verifies the validity of the modelling work.

\section{MEASUREMENT PRINCIPLE}

\section{A. Principle of Correlation Velocoty Measurement}

Since the two electrodes in the electrode pair are identical, the downstream signal $S_{2}(t)$ is similar to the upstream one $S_{1}(t)$ with a time delay [8]. The time delay $\tau_{m}$ (transit time of particles moving from the upstream electrode to the downstream one) is determined from the location of the dominant peak in the cross-correlation function $R_{12}(m)$ of the two signals:

$$
R_{12}(m)=\frac{\sum_{k=1}^{N} s_{1}(i) s_{2}(i+m)}{\left(\sqrt{\sum_{i=1}^{N} s_{1}^{2}(i)}\right)\left(\sqrt{\sum_{i=1}^{N} s_{2}^{2}(i)}\right)}
$$

where $N$ is the number of the sampling points, $M$ is the maximum number of delayed points $m(m=0,1, \ldots, M), s_{1}(i)$ and $s_{2}(i)(i=0,1,2, \ldots, N-1, \ldots, N+M-1)$ represent the discretized signals $s_{1}(t)$ and $s_{2}(t)$, respectively. Since the spacing $L$ between the two electrodes is known, the correlation velocity is derived from:

$$
v=\frac{L}{\tau_{m}}
$$

The correlation coefficient $r$, i.e. the amplitude of the dominant peak in equation (8), indicates the similarity of the two signals. It can also be used to assess the flow stability, i.e. the closer to 1 the correlation coefficient, the more stable the flow near the measurement electrodes [10].

\section{B. Area Partition of Sensor Cross-Section and Data Fusion}

It should be pointed out that the correlation velocity and correlation coefficient from one electrode pair is not the exact "mean particle velocity" or the "mean flow stability" in the pipe due to the non-uniform sensitivity distribution of the sensor, irregular velocity profile and the numerical error of correlation algorithm [8]. In order to obtain the velocity profiles of the pulverised fuel in a square-shaped pipe over the whole cross-section, the cross-sectional area is partitioned into nine zones (Fig. 7). The 12 pairs of electrodes are marked as Pair A to $\mathrm{L}$ accordingly. As shown in Fig. 7, four corner zones are marked as Zones I, III, VII, and IX, four zones along one side wall are marked as Zones II, IV, VI, and VIII, and the central zone of the cross-section is marked as Zone V.

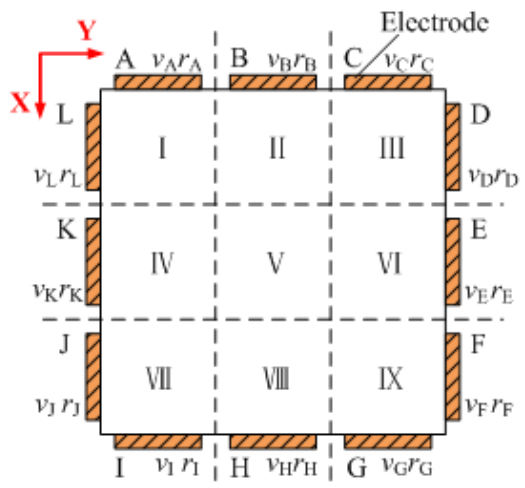

Fig. 7. Area partitions of the sensor cross-section.

A simple data fusion method is proposed in this study. For the four corner zones (Zones I, III, VII and IX), each zone has two pairs of adjacent electrodes. The velocity and correlation coefficient of each corner zone can be derived from the electrode pairs:

$$
\begin{aligned}
& v_{i}=\left(v_{j}+v_{k}\right) / 2 \\
& r_{i}=\left(r_{j}+r_{k}\right) / 2
\end{aligned}
$$

where $i=\mathrm{I}$, III, VII, IX, $j, k=A, L$, or $C, D$, or $F, G$, or $I, J$, accordingly. For Zones II, IV, VI and VIII, the velocity and correlation coefficient of each zone can be represented through the velocity and correlation coefficient of the corresponding electrode pair:

$$
\begin{aligned}
& v_{i}=v_{j} \\
& r_{i}=r_{j}
\end{aligned}
$$

where $i=\mathrm{II}, \mathrm{IV}, \mathrm{VI}, \mathrm{VIII}, j=B, E, H, K$, accordingly. For Zone V, the central zone of the cross-section, the velocity and correlation coefficient can be derived from Zones II, IV, VI and VIII, i.e.:

$$
\begin{aligned}
& v_{\mathrm{V}}=k\left(v_{\mathrm{II}}+v_{\mathrm{IV}}+v_{\mathrm{VI}}+v_{\mathrm{VIII}}\right) / 4 \\
& r_{\mathrm{V}}=k\left(r_{\mathrm{II}}+r_{\mathrm{IV}}+r_{\mathrm{VI}}+r_{\mathrm{VIII}}\right) / 4
\end{aligned}
$$

where $k$ is a dimensionless parameter, depending on the pipeline configuration (size and layout of the pipe) and flow regime (particle size, mass flow rate, air velocity, and so on) [19]. The value of the $k$ can be obtained through calibration test. Substituting equations (10)-(13) into (14)-(15) yields: 


$$
\begin{aligned}
& v_{\mathrm{V}}=k\left(v_{\mathrm{B}}+v_{\mathrm{K}}+v_{\mathrm{E}}+v_{\mathrm{H}}\right) / 4 \\
& r_{\mathrm{V}}=k\left(r_{\mathrm{B}}+r_{\mathrm{K}}+r_{\mathrm{E}}+r_{\mathrm{H}}\right) / 4
\end{aligned}
$$

\section{EXPERIMENTAL TESTS AND RESULTS}

\section{A. Test Rig and Prototype System}

A laboratory test rig with square-shaped pipe sections, as shown in Fig. 8, was designed and constructed in this study. Stable air flow is generated from an adjustable suction system and particles are fed to the rig by an adjustable vibrating feeder. The dimension of the inner side of the square-shaped pipe is 54 $\mathrm{mm}$. A prototype of sensor arrays was constructed and mounted on a horizontal pipe section (Fig. 9). A top view of the one side of the sensor arrays is illustrated in Fig. 10. As can be seen, three pairs of electrodes, made of copper, are embedded in a printed circuit board (PCB). Each electrode has a length of 15 $\mathrm{mm}$ and a width of $3 \mathrm{~mm}$, and the centre-to-centre spacing between the two adjacent electrodes in parallel is $15 \mathrm{~mm}$. The signal conditioning circuits with amplifiers and filters are placed on the other side of the PCB and the cut-off frequency of the filter is set as $18 \mathrm{kHz}$.

\section{B. Test Programme}

Since the size and shape of the coal and flour particles are similar, flour is used as a substitute of pulverised coal for health reasons under laboratory conditions. The mean diameter and bulk density of the flour particles are about $150 \mu \mathrm{m}$ and 0.52 $\mathrm{g} / \mathrm{cm}^{3}$, respectively. Experiments were conducted on the square-shaped particle flow test rig under nine test conditions: three different air-velocities (marked as $v 1, v 2$, and $v 3$ ) with three different mass flow rates (marked as $Q 1, Q 2$, and $Q 3$ ), as shown in Table I. The ambient temperature was around $26^{\circ} \mathrm{C}$ whilst the relative humidity was $42 \%$ during the test period.

A hot-wire anemometer (Model TPI 575) was used to measure the pure air velocities in the nine zones of the pipe cross section. Since the temperature and relative humidity in the laboratory ware constant and the flour was conveyed in a dilute phase suspension, parameter $k$ used to characterise the flour flow in Zone $\mathrm{V}$ was determined by dividing the velocity of pure air in Zone V by the mean velocity of air in Zones II, IV, VI and VIII under three different air-velocity conditions $v 1, v 2$, $v 3$ (Table I). The resulting value of $k$ is 1.07 .

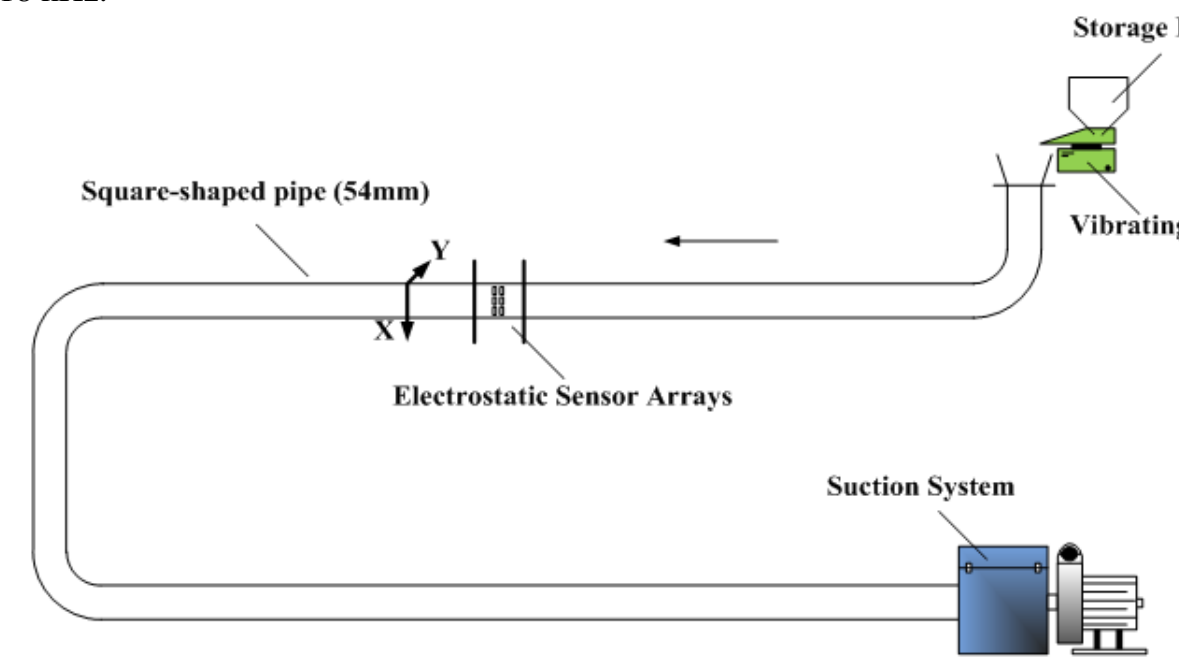

Fig. 8. Layout of the pneumatic conveying test rig.

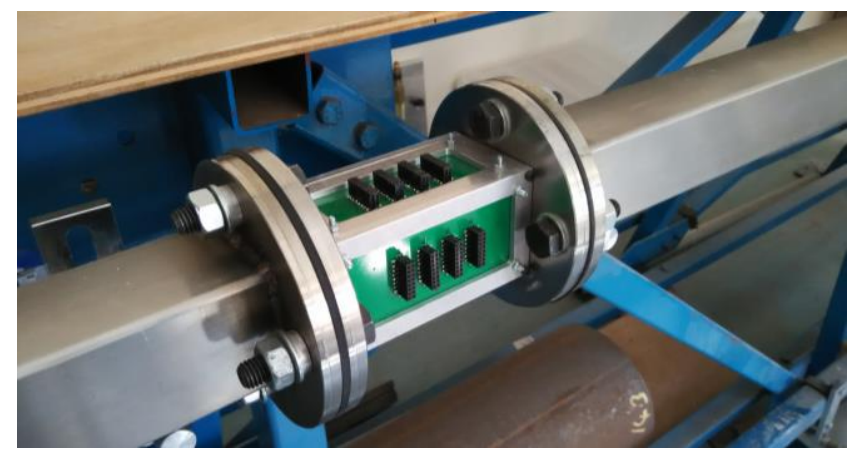

Fig. 9. Sensing head under test on the horizontal square-shaped test pipe section.

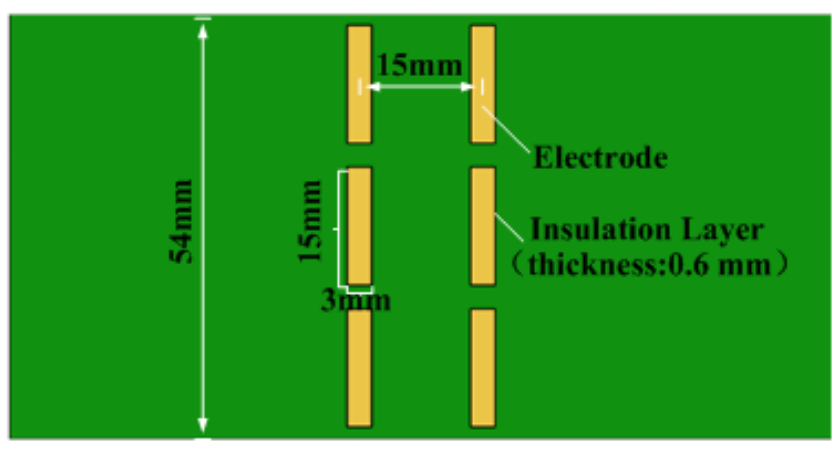

Fig. 10. Dimensions of the electrostatic sensor arrays.

TABLE I

TEST PROGRAMME

\begin{tabular}{ccccccccc}
\hline \hline & \multicolumn{3}{c}{ Air Velocities (m/s) } & \multicolumn{3}{c}{ Mass Flow Rate $(\mathrm{kg} / \mathrm{h})$} & \multicolumn{3}{c}{ Mean Volumetric Concentration (\%) } \\
$v 1$ & $v 2$ & $v 3$ & $Q 1$ & $Q 2$ & $Q 3$ & $\beta_{1}($ in $Q 1)$ & $\beta_{2}($ in $Q 2)$ & $\beta_{3}($ in $Q 3)$ \\
\hline $11.0-12.6$ & $11.5-13.7$ & $12.8-14.5$ & 5.0 & 10.6 & 14.6 & 0.073 & 0.155 & 0.214 \\
\hline \hline
\end{tabular}




\section{Experimental Results and Discussion}

Experimental work under each test condition was repeated for three times and, for each pair of electrodes, 2048 data samples from the upstream and downstream signals were recorded for each test cycle. A total of 1500 readings for the velocity and correlation coefficient were taken from one pair of electrodes under each test condition. Two typical signals from a pair of electrodes (air velocity $v 2$, mass flow rate of particles $Q 1$, and sampling rate of $50 \mathrm{kHz}$ ) and their corresponding cross-correlation function are illustrated in Fig. 11, where $s_{1}(t)$ and $s_{2}(t)$ are the upstream and downstream signals, respectively.

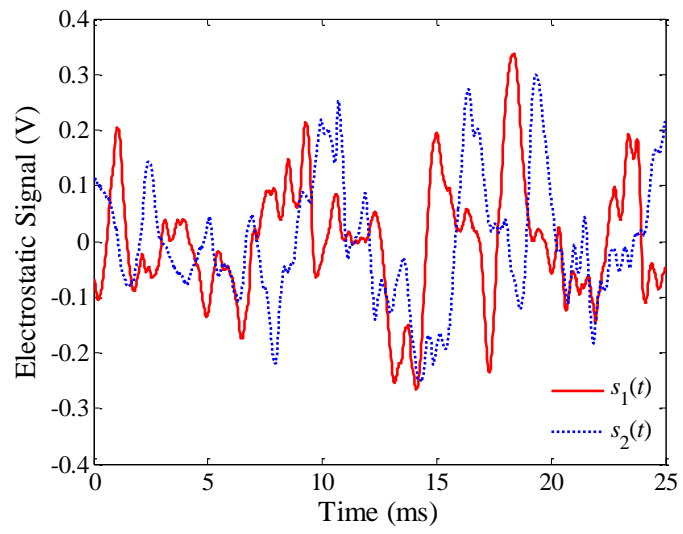

(a) Signals from a pair of electrodes

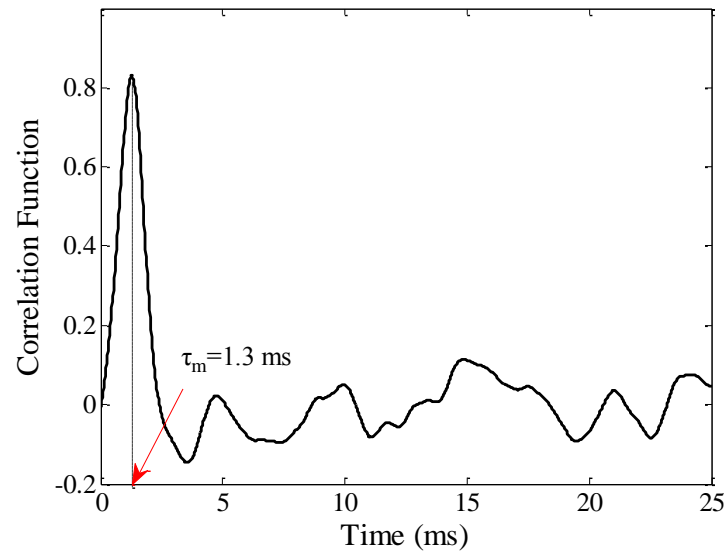

(b) Cross-correlation function of the two signals in (a)

Fig. 11. Signals from one electrode pair and corresponding cross-correlation function.

The velocity profiles of pure air (measured by the hot-wire anemometer TPI 575) with three different air velocities $v 1, v 2$, and $v 3$ are shown in Fig. 12. The three zones (Zones I, II, and III) are on the upside and another three zones (Zones VII, VIII, and IX) are on the downside. The profiles clearly show that the air velocities in the nine zones are non-uniform, i.e., the air velocity in the centre of the pipe (Zone V) is the highest and the air velocity in the four zones (Zones II, IV, VI and VIII) is higher than that in the four corner zones (Zones I, III, VII and IX). This is because more friction exists on the fluid boundary layer of the air and the wall in the areas near the pipe wall.
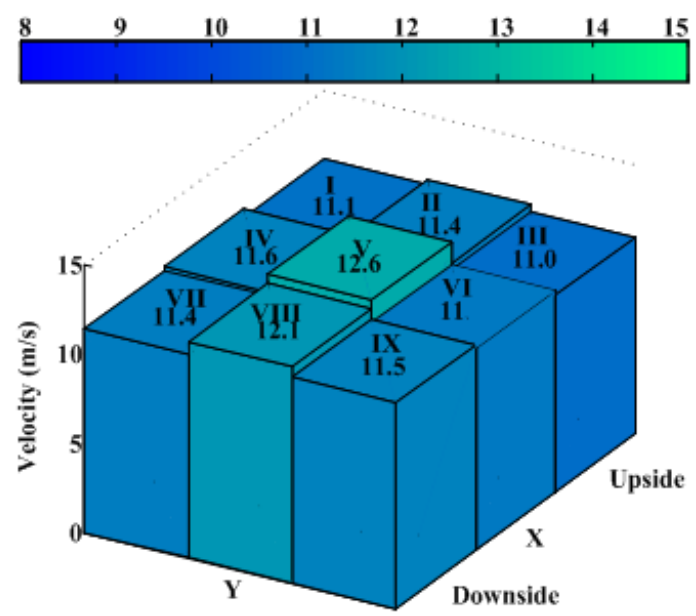

(a) Air velocity $v 1$

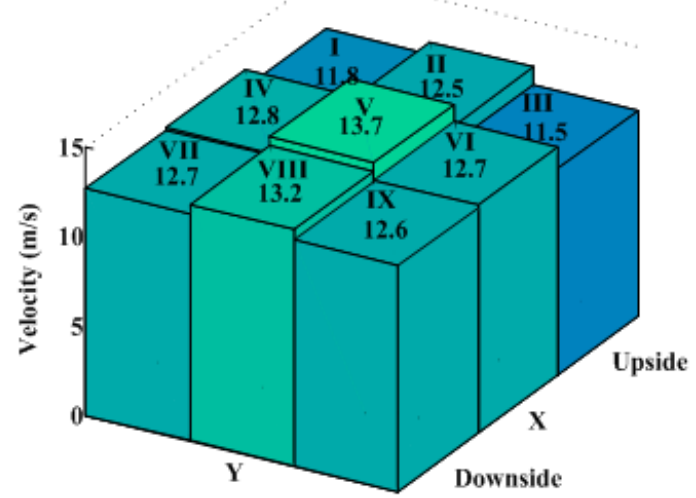

(b) Air velocity $v 2$

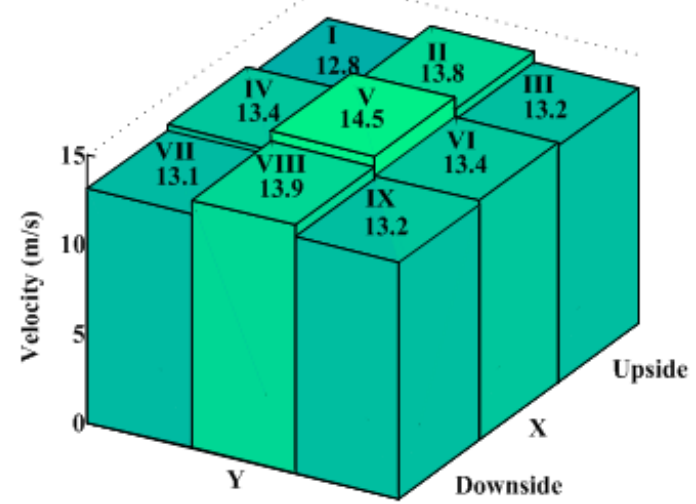

(c) Air-velocity $v 3$

Fig. 12. Air velocity profiles.

The velocity profiles of particles for the three different mass flow rates $Q 1, Q 2$, and $Q 3$ under the three air velocities $v 1, v 2$, and $v 3$ are plotted in Fig. 13 to Fig. 15. The color bars used in Fig. 12 to Fig. 15 are the same as in Fig. 11. As excepted, the particles in the central zone (Zone V) move at a higher velocity than those in the zones with one side pipe wall adjacent (Zones II, IV, VI and VIII) and the particles in the corner zones (Zones I, III, VII and IX) travel the most slowly. This is because the particles near the pipe wall experience more friction due to the interactions between the wall, air, and particles. Meanwhile, though the sensor arrays are mounted on the horizontal pipe section (Fig. 9), obvious velocity difference between the 
particles in the three upper zones (Zones I, II, and III) and those in the three bottom zones (Zones VII, VIII, and IX) is not evident in Fig. 13 - Fig. 15. Such phenomenon means that the gravitational effect of the particles conveyed in a dilute phase flow is relatively small. Furthermore, the measured velocity profiles of particles in the nine test conditions are very similar, demonstrating that the electrostatic sensor arrays have a good repeatability.

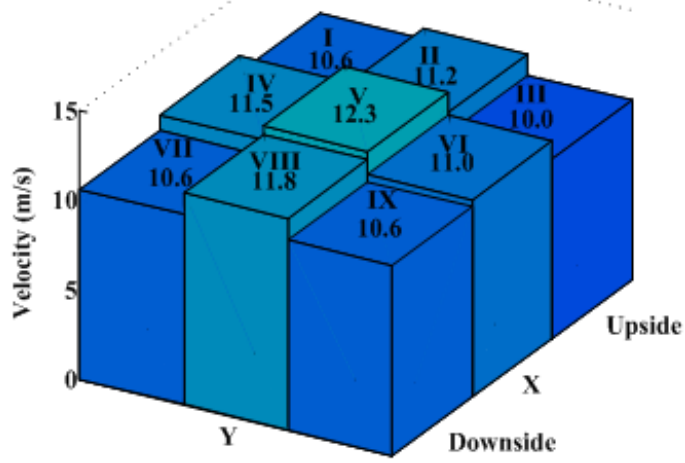

(a) Mass flow rate $Q 1$

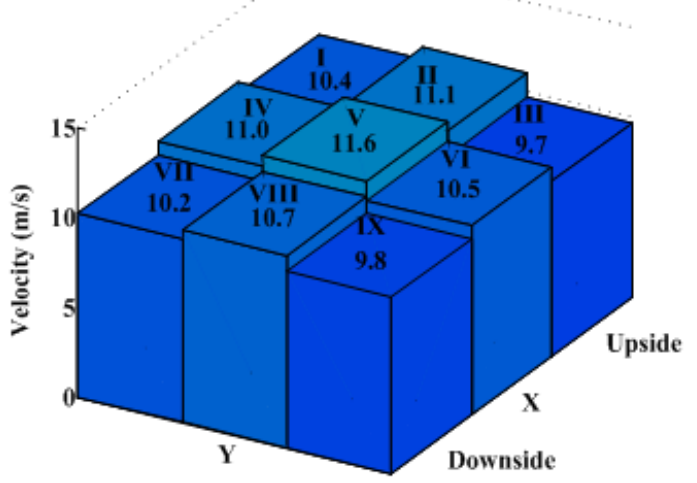

(b) Mass flow rate $Q 2$

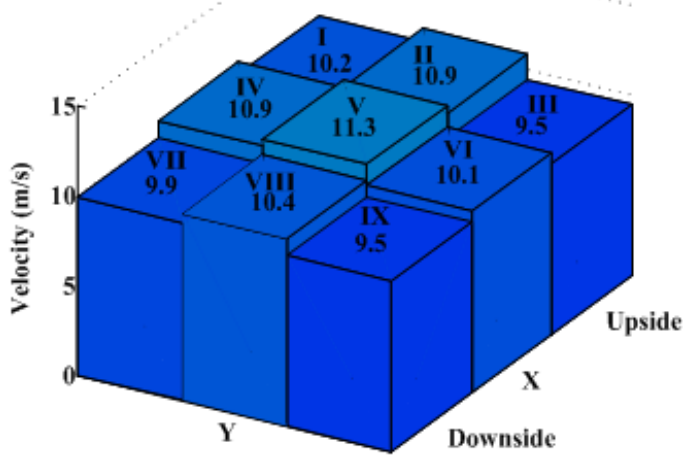

(c) Mass flow rate $Q 3$

Fig. 13. Velocity profiles of particles with air velocity v1.

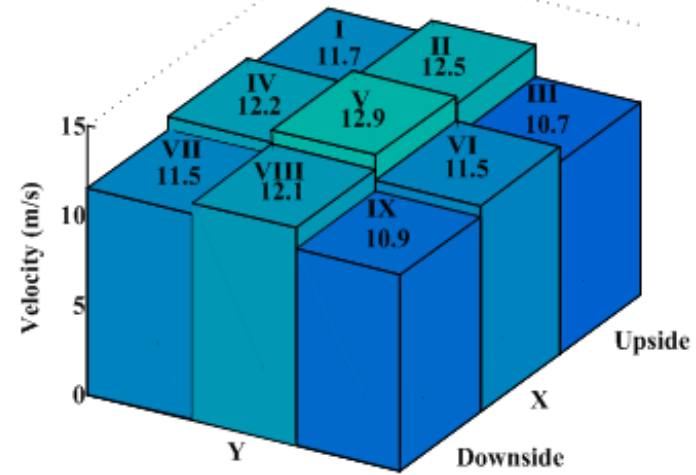

(a) Mass flow rate $Q 1$

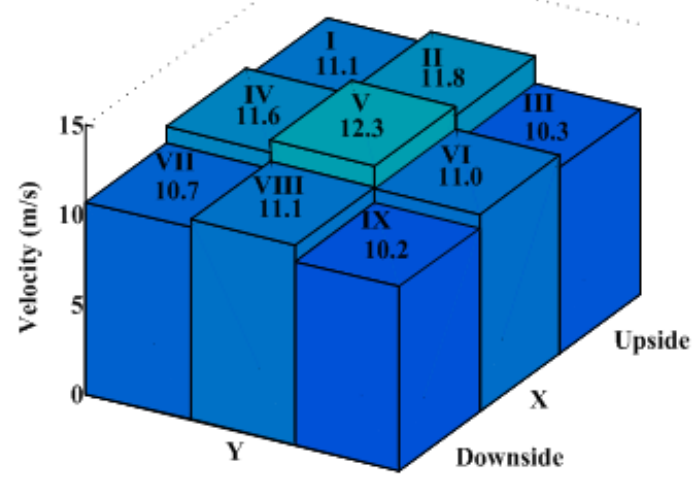

(b) Mass flow rate $Q 2$

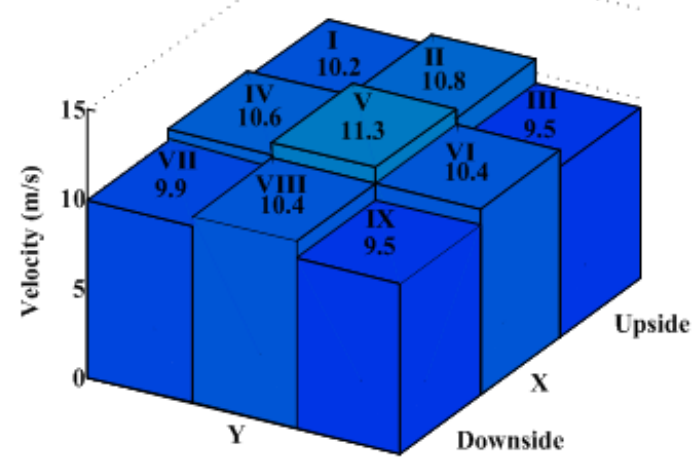

(c) Mass flow rate $Q 3$

Fig. 14. Velocity profiles of particles with air velocity v2.

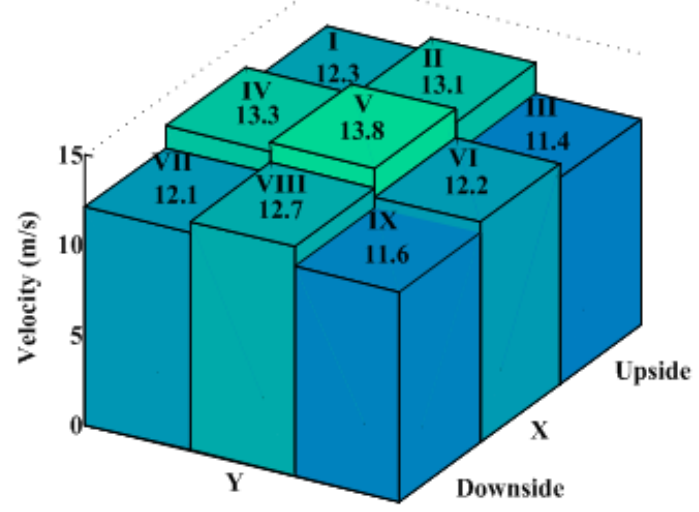

(a) Mass flow rate $Q 1$ 


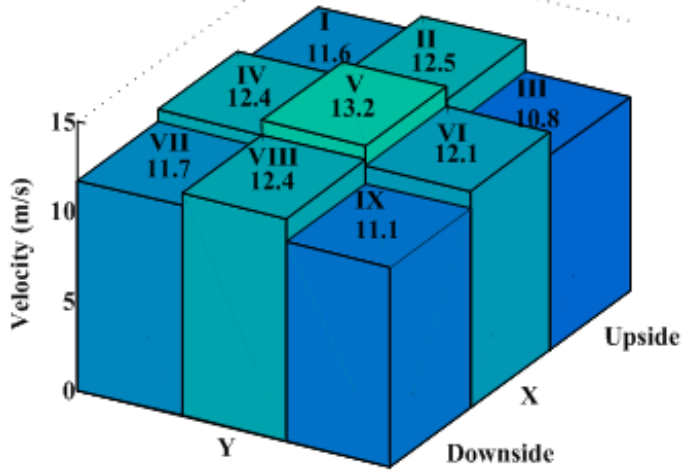

(b) Mass flow rate $Q 2$

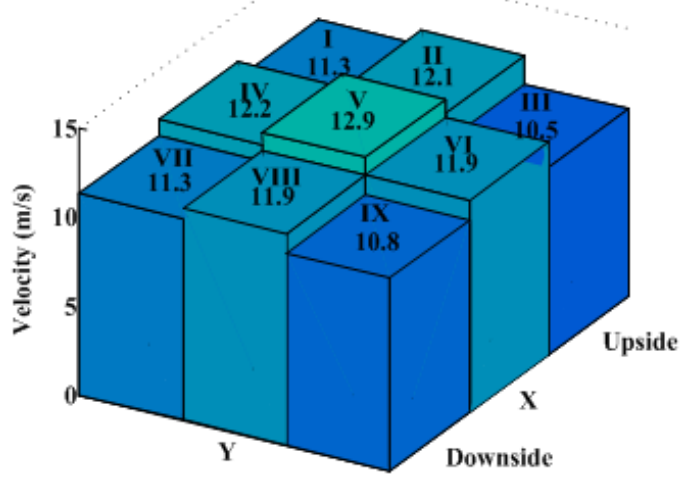

(c) Mass flow rate $Q 3$

Fig. 15. Velocity profiles of particles with air velocity v3.

By comparing Fig. 12 to Fig. 15, it can be seen that the velocity profiles of particles over the whole pipe cross section are similar to that of air but the particles travel slower due to the drag force exerted on the particles [19]. In addition, for a constant air velocity $v_{i}(i=1,2$, or 3$)$, the particle velocity decreases as the mass flow rate of particles increases. For a constant mass flow rate $Q_{i}(i=1,2$, or 3$)$, the particle velocity increases with the air velocity. The slip velocity between the air and particles over the cross section of the pipe under the nine test conditions are shown in Fig. 16. The slip velocity distribution across the pipe section demonstrates that the slip velocity increases with the mass flow rate of particles or air velocity. Besides, the slip velocity at the bottom zones (Zones VII, VIII, and IX) is greater than that at the upper zones (Zones I, II, and III) due to the gravitational effect.

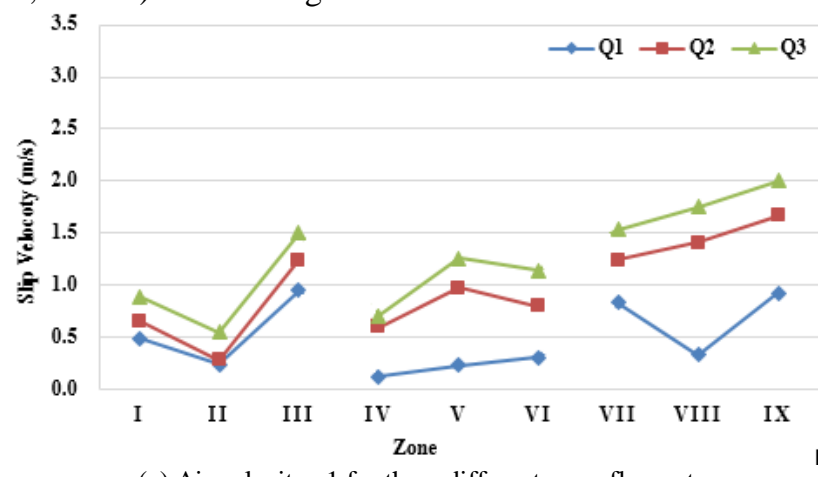

(a) Air velocity $v 1$ for three different mass flow rates

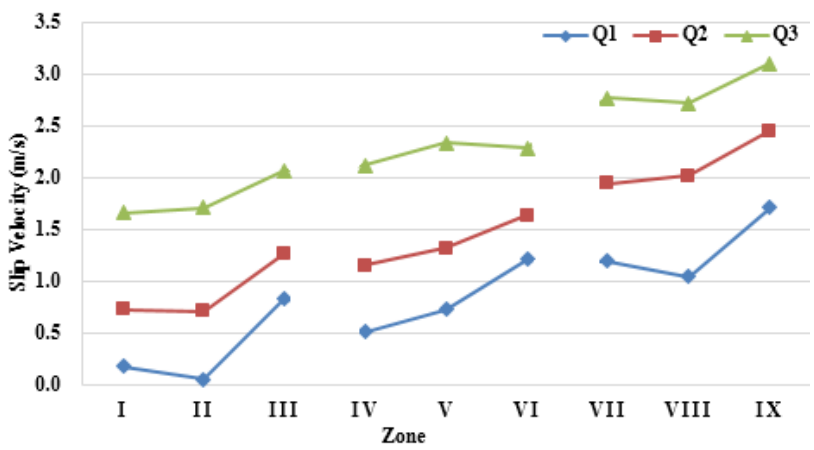

(b) Air velocity $v 2$ for three different mass flow rates

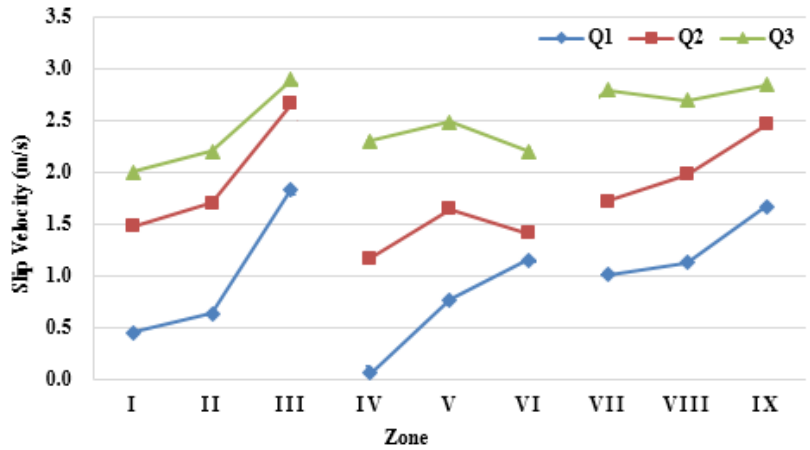

(c) Air velocity $v 3$ for three different mass flow rates

Fig. 16. The slip velocity between the air and particles over the cross section.

The velocity fluctuations of the air and particles over the whole cross-section can be reflected by the standard deviations (SD) of the measured velocities among the nine zones, i.e., the larger the SD, the higher the fluctuation. Meanwhile, the fluctuations in velocities can also represent the stability of the flow, that is, the less fluctuations, the more stable the flow. The SDs of the measured air and particle velocities of the nine zones (depicted in Fig. 12 to Fig. 15) are shown in Fig. 17. Each data point illustrated in Fig. 17 uses the nine velocity values (each particles velocity is obtained by averaging more than 1500 readings) under each test condition to calculate the standard deviation. It is obvious that, the SDs of the particle velocities are higher than those of air because the particle flow has more complex behavior than the air flow. Meanwhile, it is observed that the SD of particle velocities decreases as the mass flow rate of particles increases or as the air velocity decreases. Such phenomena reflect that the flow is more stable in relatively higher concentration conditions.

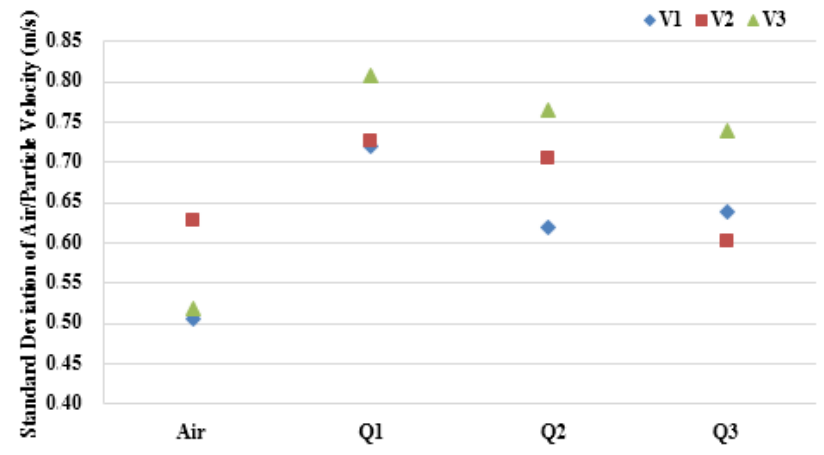

Fig. 17. Standard deviation of the measured air and particle velocities.

The correlation coefficient of the particle flow under the nine test conditions are shown in Fig. 18. For the whole 
cross-section of the square-shaped pipe, it is clear that the correlation coefficient in the centre (Zone IV) is higher than those in Zones II, IV, VI and VIII and the correlation coefficient in the corner zones (Zone I, III, VII and IX) is the smallest, indicating that the particles in the centre of the pipe move more stably and the dynamics of the flow in the corners is more complex. These observations are consistent over all the nine test conditions. Meanwhile, we can observe that, for the higher air velocities ( $v 2$ and $v 3$ ), the correlation coefficient increases with the mass flow rate of particles, which also suggests that the flow is more stable under higher mass flow rate conditions.

The flow stability among the nine zones can also be reflected by the standard deviation of the correlation coefficient, i.e. the smaller the standard deviation of the correlation coefficient, the more stable the flow. As illustrated in Fig. 19, the standard deviation of the correlation coefficient decreases as the mass flow rate of particles increases for the higher air velocities $(v 2$ and v3), demonstrating that the flow over the whole cross-section is more stable under a higher mass flow rate of particles. However, for the lower air velocity $(v 1)$, this observation does not hold true because the particles may not be fully suspended. Such a trend also agrees well with the standard deviation of the measured velocities (Fig. 17).

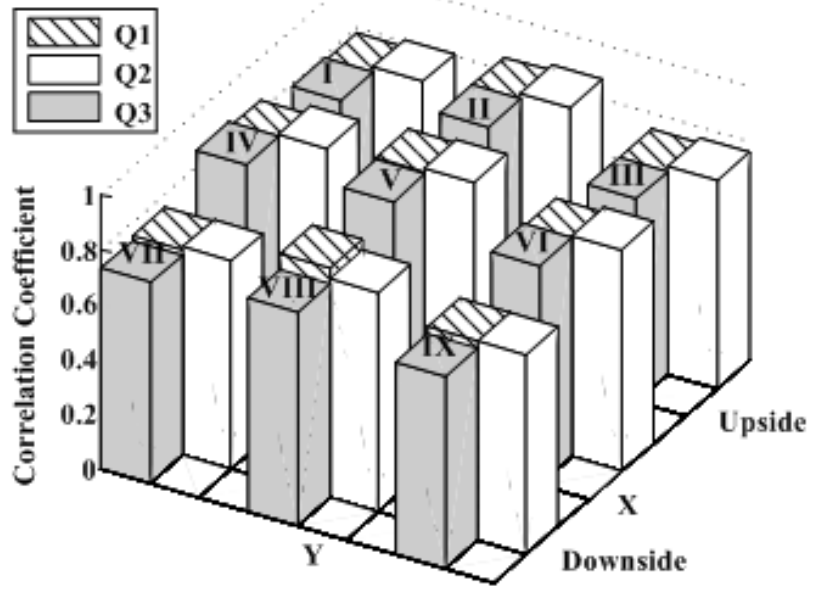

(a) Air velocity $v 1$ for three different mass flow rates

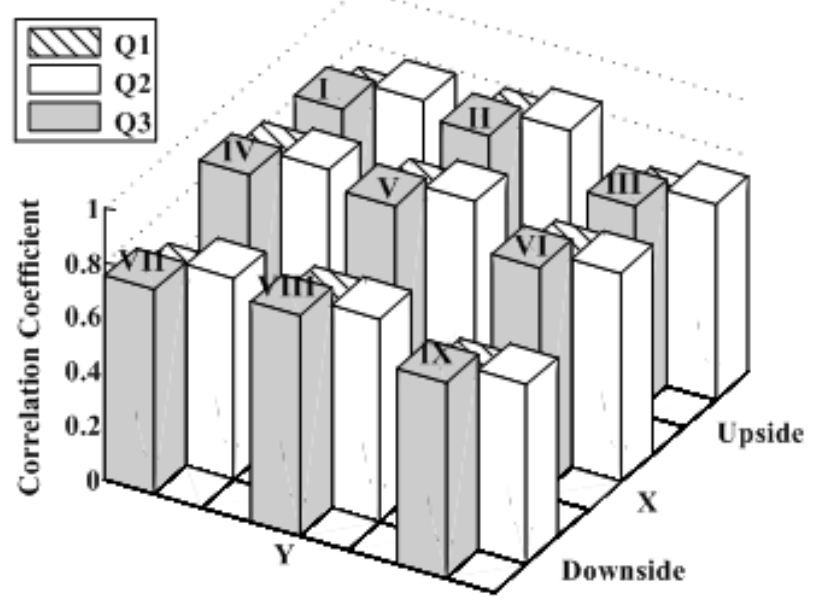

(b) Air velocity $v 2$ for three different mass flow rates

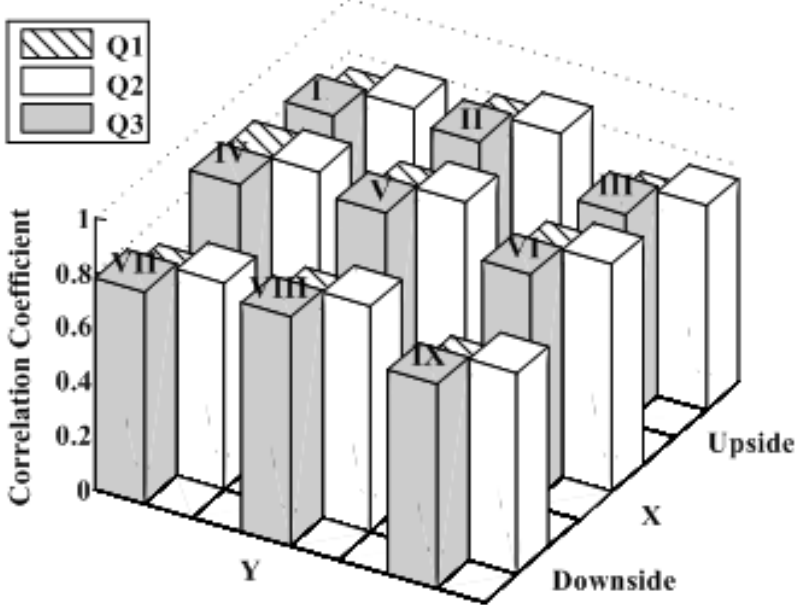

(c) Air velocity $v 3$ for three different mass flow rates

Fig. 18. Correlation coefficients under the nine different test conditions.

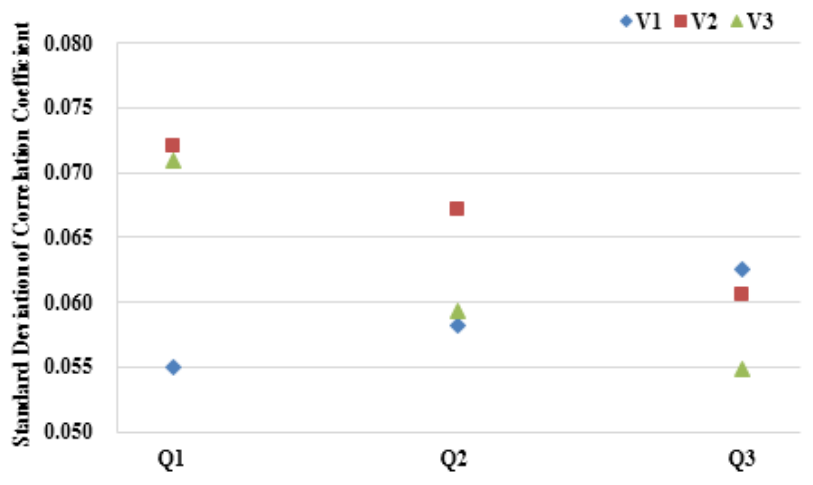

Fig. 19. Standard deviation of the correlation coefficients over the whole cross-section.

\section{CONCLUSIONS}

The non-restrictive electrostatic sensor arrays used to characterise pulverised fuel flow in a square-shaped pneumatic conveying pipe have been designed, mathematically analyzed, constructed and tested. A mathematical model of the electrostatic sensor arrays has been established by applying the ideal point charge concept and the spatial sensitivity distribution, induced charge and currents of different electrodes based on the model has been derived. Meanwhile, experimental tests were undertaken on a laboratory-scale test rig under nine different test conditions. Several conclusions can be drawn from the modelling and experimental results presented in this paper. First, the non-restrictive electrostatic sensor arrays with distributed strip-shaped electrodes are capable of characterising the velocity profiles of pulverised fuel in a square-shaped pipe. The velocity profiles of air and particles over the whole cross-section of the pipe are similar and relatively non-uniform. Second, the slip velocity between the air and particles ranges between $0.1 \mathrm{~m} / \mathrm{s}$ (low air velocity $v 1$ with low mass flow rate $Q 1$ ) and $2.9 \mathrm{~m} / \mathrm{s}$ (high air velocity $v 3$ with high mass flow rate Q3). Meanwhile, the slip velocity increases with the mass flow rate of particles or air velocity. Finally, the particles in the central area of the pipe travel faster and are more stable than those in other zones whilst the particles in the four corners are the slowest and most unstable because of the confined space of the corners. 


\section{REFERENCES}

[1] S. Liu, Q. Chen, H. Wang, F. Jiang, I. Ismail, and W. Yang, "Electrical capacitance tomography for gas-solids flow measurement for circulating fluidized beds," Flow Meas. Instrum., vol. 16, no. 2, pp. 135-144, 2005.

[2] Y. Zheng, D. McGlinchey, J. Pugh, and Y. Li, "Experimental investigation on heat transfer mechanisms of pneumatically conveyed solids' plugs as a means to mass flow rate measurement," Flow Meas. Instrum., vol. 40, pp. 232-237, 2014.

[3] Y. Zheng, J. R. Pugh, D. McGlinchey, and R. O. Ansell, "Simulation and experimental study of gas-to-particle heat transfer for non-invasive mass flow measurement," Measurement, vol. 41, no. 4, pp. 446-454, 2008.

[4] S. J. Tallon, and C. E. Davies, "The effect of pipeline location on acoustic measurement of gas-solid pipeline flow," Flow Meas. Instrum., vol. 11, no. 3, pp. 165-169, 2000.

[5] M. Anklin, W. Drahm, and A. Rieder, "Coriolis mass flow meters: overview of the current state of the art and latest research," Flow Meas. Instrum., vol. 17, no. 6, pp. 317-323, 2006.

[6] X. Cai, J. Li, X. Ouyang, Z. Zhao, and M. Su, "In-line measurement of pneumatically conveyed particle by a light transmission fluctuation method," Flow Meas. Instrum., vol. 16, no. 5, pp. 315-320, 2005.

[7] Y. Zheng, and Q. Liu, "Review of techniques for the mass flow rate measurement of pneumatically conveyed solids," Measurement, vol. 44, no. 4, pp. 589-604, 2011.

[8] Y. Yan, B. Byrne, S. Woodhead, and J. Coutlhard, "Velocity measurement of pneumatically conveyed solids using electrodynamic sensors," Meas. Sci. Technol. vol. 6, no. 5, pp. 515-537, 1995.

[9] M. Machida, and B. Scarlett, "Process tomography system by electrostatic charge carried by particles,"IEEE Sens. J., vol. 5, no. 2, pp. 251-259, 2005.

[10] X. Qian, Y. Yan, J. Shao, L. Wang, H. Zhou, and C. Wang, "Quantitative characterization of pulverised coal and biomass-coal blends in pneumatic conveying pipelines using. electrostatic sensor arrays and data fusion techniques," Meas. Sci. Technol., vol. 23, no. 8, 085307 , 2012.

[11] Z. Kan, X. Wang, and S. Zhang, "Study on the spatial filtering and sensitivity characteristic of inserted electrostatic sensors for the measurement of gas-solid two-phase flow parameters," Flow Meas. Instrum., vol. 30. pp. 26-33, 2013.

[12] C. Xu, J. Li, and S. Wang, "A spatial filtering velocimeter for solid particle velocity measurement based on linear electrostatic sensor array," Flow Meas. Instrum., vol. 26. pp. 67-78, 2012.

[13] J. B. Gajewski, "Electrostatic nonintrusive method for measuring the electric charge, mass flow rate, and velocity of particulates in the two-phase gas-solid pipe flows-its only or as many as 50 years of historical evolution. IEEE Trans. Ind. Appl., vol. 44, pp. 1418-1430, 2008.

[14] S. N. Murnane, R. N. Barnes, S. R. Woodhead, and J. E. Amadi-Echendu, "Electrostatic modelling and measurement of airborne particle concentration," IEEE Trans. Instrum. Meas., vol. 45, no. 2, pp. 488-492, 1996.

[15] L. Peng, Y. Zhang, and Y. Yan, "Characterization of electrostatic sensors for flow measurement of particulate solids in square-shaped pneumatic conveying pipelines," Sensor Actuat. A-Phys., vol. 141, no. 1, pp. 59-67, 2008.

[16] J. Yao, Y. Zhao, and M. Fairweather, "Numerical simulation of turbulent flow through a straight square duct," Appl. Therm. Eng., vol. 91, pp. 800-811, 2015.

[17] J. Adams, M. Fairweather, and J. Yao, "Modelling and simulation of particle re-suspension in a turbulent square duct flow," Comput. Chem. Eng., vol. 35, no. 5, pp. 893-900, 2011.
[18] D. J. Griffiths, "Introduction to Electrodynamics," Addison-Wesley, 4th ed., pp. 124-126, 2012.

[19] D. Mills, "Pneumatic Conveying Design Guide," Butterworth-Heinemann Pub., 2nd ed., Oxford, U.K., pp. 236-257, 2004. 